\title{
Development of a diet quality index for the Scottish population
}

\author{
K.L. Barton ${ }^{1}$, W.L. Wrieden ${ }^{2}$, L.F. Masson ${ }^{3}$, A.S. Anderson ${ }^{4}$, A. Sherriff ${ }^{5}$ and J.A. Armstrong ${ }^{6}$ \\ ${ }^{1}$ Division of Food and Drink, Abertay University, Dundee DD1 $1 \mathrm{HG}$, \\ ${ }^{2}$ Human Nutrition Research Centre and Institute of Health and Society, Newcastle University, Newcastle upon Tyne \\ NE2 $4 H H, U K$, \\ ${ }^{3}$ School of Pharmacy and Life Sciences, Robert Gordon University, Aberdeen, AB10 7GJ, \\ ${ }^{4}$ Centre for Public Health Nutrition Research, University of Dundee, Dundee, UK, \\ ${ }^{5}$ School of Medicine, Dentistry and Nursing, University of Glasgow, Glasgow, UK and \\ ${ }^{6}$ School of Health and Life Sciences, Glasgow Caledonian University, Glasgow, UK
}

Secondary analysis of food purchase data has been used to monitor progress towards Scottish Dietary Targets $(\text { Goals })^{(1)}$ since $2001^{(2)}$, however little work has been carried out to assess the overall quality of the diet of the Scottish population. A diet quality index (DQI) is a useful tool that provides an overall rating of an individual's dietary intake in reference to nutrient and/or dietary recommendations $^{(3)}$ or a diet pattern that is considered to be beneficial to health ${ }^{(4)}$. Numerous diet quality indices have been created as summary measures of diet, but to our knowledge, none are available that include all of the Scottish Dietary Goals ${ }^{(1)}$.

The purpose of this work was to explore the feasibility of developing a DQI to be used with food purchase data to assess the quality of the Scottish Diet. A scoring system was devised (originally in collaboration with the advisors from Food Standards Scotland) which provides details of the foods and nutrients that were included in the DQI and the scoring methodology and rationale for each component. The definitive index comprised three food scores (fruit and vegetables; oil rich and white fish; and red and processed meat), and six nutrient scores (fat; saturated fat; intrinsic and milk sugars and starch; non-milk extrinsic sugars; non-starch polysaccharides; and alcohol) with a total score out of 85. For ease of interpretation, this was converted to a percentage score. Annual household food purchase data from 2001 to 2012, for Scotland, from the UK Living Costs and Food Survey were analysed to estimate the mean DQI of the Scottish population. Adjustments were made to allocate the correct proportion of each food to the appropriate food group (including to composite foods), for waste ${ }^{(5)}$, and for weight increase or loss due to cooking or dilution ${ }^{(6)}$. Data were analysed using general linear models within the complex samples module of SPSS (SPSS Inc., Chicago, IL, USA) weighting to the Scottish population and taking account of sampling methods.

The DQI was distributed across the population, the mean for the combined period of 2001 to 2012 was $36.5 \%$ (95\%CI $36.0-37.0 \%$ ). Only a small proportion (less than $1 \%$ ) of the population scored highly indicating adherence to most of the targets. DQI increased slightly but significantly between 2001 and 2012 from $35.5 \%(95 \%$ CI $34.0-37.0 \%)$ in 2001 to $36.6 \%$ (95\%CI $35.3-37.8 \%$ ) in 2012 (p-value for linear association $<0.001$ ). Positive Spearman's correlations were found between the DQI score, and folate $(\mathrm{r}=0.448 ; \mathrm{p}<0.001)$ and vitamin $\mathrm{C}$ intakes $(\mathrm{r}=0.331 ; \mathrm{P}<0.001)$ (per 2000kcal $(8368 \mathrm{~kJ})$ ) indicating that the DQI was positively associated with other aspects of the diet.

The DQI reported here provides a pragmatic way of assessing the quality of the diet purchased by the Scottish and other populations. The low scores obtained for the majority of the population are in part due to the score / no score policy for meat and meat products, macronutrients and alcohol. This meant that a household could obtain zero for a food or nutrient score despite being close to meeting the target. However it was felt that using sliding scales to another arbitrary value for foods and nutrients targeted for decrease was not desirable. The score provides an indication of the quality of the diet based on current dietary guidelines, which can be assessed for relationships with socioeconomic status, household composition and the environmental impact of dietary choices.

Funded by Food Standards Scotland (S14034 / FS424018). Data from DEFRA, SNS, ONS and the UK Data Archive.

1. Scottish Government (2013) Revised Dietary Goals for Scotland. Edinburgh: Scottish Government.

2. Wrieden WL, Armstrong J, Sherriff A et al. (2013) BJN, 109, 1892-1902.

3. Wirt A and Collins CE (2009) Public Health Nutr, 12(12), 2473-2492.

4. Panagiotakos DB, Pitsavos C, and Stefanadis C. (2006) Nutr Metab Cardiovasc Dis 16(8), 559-568.

5. Waste and Resource Action Programme (2007) The food we waste. Oxon: WRAP.

6. Barton KL, Wrieden WL, Sheriff A et al. (2016) Public Health Nutr, 18, 2970-2980. 